\title{
Experimental Demonstration of the Impact of the Fringe Shape in Sub-Lambda / 2 Sensing With Optical Feedback Interferometry
}

\author{
Einar Knudsen ${ }^{1,2}{ }^{*}$, Julien Perchoux ${ }^{1}$, Thierry Mazoyer ${ }^{2}$, José Imas ${ }^{1}$, Mengkoung Veng ${ }^{1}$, \\ Francis Jayat ${ }^{1}$, ClÉment TronChe ${ }^{1}$, AND Thierry Bosch ${ }^{1}$ \\ ${ }^{1}$ LAAS-CNRS, Université de Toulouse, CNRS, INP, 31062 Toulouse, France \\ ${ }^{2}$ ACOEM, Department of Innovation, 200 Allée des Ormeaux, Limonest, France \\ *Corresponding author: einar.knudsen@toulouse-inp.fr, einar.knudsen@acoem.com
}

Compiled November 24, 2020

\begin{abstract}
It is established in the Optical Feedback Interferometry (OFI) theory that the shape of the interferometric fringe has an impact on the detector's response to very small displacement measurements. In this paper we validate - for the first time based on experimental results - this statement by comparing experiments to an established model implementation. Through these experiments we show that the amplitude of the signals induced by sub-lambda/2 optical path variations is linearly dependent on the slope of the underlying fringe. Thus, careful control of the phase allows us to maximize the detection amplitude of very small displacements by positioning the phase where the fringe slope is the steepest. These results are directly applicable to established OFI applications that measure sub-lambda/2 optical path variations, such as OFI vibrometers or acoustic imaging though the acousto-optic effect. ๑ 2020 Optical Society of America
\end{abstract}

http://dx.doi.org/10.1364/ao.XX.XXXXXX

\section{INTRODUCTION}

Optical Feedback Interferometry (OFI) is a compact and versatile non-contact sensing scheme $[1,2]$ with numerous areas of application. It is used in displacement sensing [3-5] including vibrometry [6-8], absolute distance measurements [9-11], velocimetry $[12,13]$ and flowmetry [14-16], to mention a few. OFI is compact, robust, self-aligned and above all, it's need for very few optical elements compared to classical interferometric devices makes it a low cost sensing scheme. Recent advances [17-19] have made the technology capable of measuring displacements in the range of $120 \mathrm{pm}$ which is a major improvement of the OFI sensing scheme. This approach requires additional optical elements, and the increase of system complexity makes this approach cumbersome as it approaches the number of elements used in commercial interferometers today.

Nevertheless, the OFI sensing scheme gains importance in various sensing domains such as vibrometry or the very promising acoustic imaging through the acousto-optical effect. Bertling et al [20] and Urgiles et al [21] created visual representations of sound waves in air using OFI. Their method is based on the sensing of the acousto-optic effect, where acoustic pressure variations induce modulations of the refractive index of air. These minute modulations create small changes in the optical path between the laser and the target. The variations are far below a half laser wavelength and at the edge of the limitations of the technology in it's classical compact form.

OFI operates the following way: Coherent light from the laser is backscattered from a target and returns back into the laser cavity. The returning light is coherently mixed with the lasing field. The mixing induces perturbations in the laser wave phase and amplitude, the latest which can be measured by the laser's in-package photodiode. An analysis of the signal provides information about the target it is pointing on. In particular, if the target is moving, interferometric fringes corresponding to a displacement of half the wavelength $\lambda$ will be observed. By simply counting fringes we can record the target's movement along the beam axis.

Ameliorations were made to the OFI sensing scheme by Giuliani et al [6] to improve the lower limits of detection in vibrometry applications. They devised a non-contact vibrometer where the system was designed to maintain the laser phase at an operating point through an electronic feedback loop, a method called phase locking. In Giuliani's approach the operating point is set to the half fringe, as it allows a larger measurement dynamic. Measurements of down to $0.1 \mathrm{~nm}$ are reported. The advantage of this system was that there would be no drift in signal amplitude for a given measurement due to changes in optical conditions.

As explained by Taimre et al [2], for sub- $\lambda / 2$ displacements, the target's movement is directly reproduced in the interferometric signal in an analog fashion. Lukashkin et al [22], inspired 
by the approach in [6], regulated the mean distance between the laser and an oscillating target in order to fix an operating point around which the system would operate. This served to reduce distortions of the signal due to the fringe shape.

The shape of the fringe has an impact on measurements of sub- $\lambda / 2$ optical path variations. A deeper knowledge of these effects would be beneficial for the further development of systems such as Giuliani's. The detection capacities of these types of OFI systems could be ameliorated by locking the phase at a point where the fringe slope is steeper than on the half-fringe point. This would increase the signal gain amplitude for a given excitation.

Experiments coupled with a comprehensive model has not yet been performed to explicitly show the impact of the fringe shape on the phase-to-power conversion for small displacements.

In this paper we present a dedicated, repeatable and robust experiment showing the impact of the fringe shape on small signals. We demonstrate experimentally that the signal amplitude is linearly coupled to the slope of the fringe, a result not yet explicitly published in the OFI literature. Furthermore we model an OFI system measuring sub- $\lambda / 2$ optical path variations using Kliese's algorithm [23]. Finally, we show the algorithm's performance in simulating sub- $\lambda / 2$ optical path variations through accurately simulating the experimental results.

\section{THEORY: OFI MODEL AND GOVERNING EQUATIONS}

One of the most frequently used models for the OFI sensing scheme was made by Lang et Kobayashi [24] using modified laser rate equations. The model was simplified by Petermann [25], supposing quasi static conditions, and later written into a concise algorithm in [23]. The algorithm uses the Three Mirror Model $[2,11,25]$ as a base. This model is an extension of the two mirror laser model in which a phase condition is satisfied: the optical field in the laser has the same phase after one round trip $[2,23]$. In the Three Mirror Model, the laser is forced to change its emission frequency $v_{0}$ under feedback in order to satisfy the phase condition. The phase of the light returning into the laser is calculated through the excess phase equation [2,11,23].

$$
\phi_{\mathrm{F}}-\phi_{0}+C \sin \left(\phi_{\mathrm{F}}+\arctan \alpha\right)=0
$$

$\phi_{\mathrm{F}}=2 \pi v \tau_{\text {ext }}$ is the phase response which is the external roundtrip phase at the perturbed frequency. $\phi_{0}=2 \pi v_{0} \tau_{\text {ext }}$ is the phase stimulus which is the external round trip phase of the free running laser [2]. $v_{0}$ is the laser's running emission frequency, $v$ is the perturbed laser frequency and $\alpha$ is the linewidth enhancement factor [26]. The feedback parameter $C$ describes the coupling strengh between the laser and the external cavity $[2,27,28]$, and can be written as

$$
C=\frac{\tau_{\text {ext }}}{\tau_{\text {in }}} \epsilon \sqrt{\frac{R_{\text {ext }}}{R_{2}}}\left(1-R_{2}\right) \sqrt{1+\alpha^{2}} .
$$

Here $\tau_{\text {in }}$ is the laser cavity round-trip time for a photon, $\tau_{\text {ext }}$ the external cavity round-trip time. $R_{2}$ is the reflectivity of the front facet of the laser, $R_{\text {ext }}$ is the ratio of the reinjected over the emitted power and $\epsilon$ is the fraction of reflected light coupled coherently back into the laser [23, 27].

In our model we use the solver in [23] to compute the excess phase equation eq. (1), and thus calculate the laser power eq. (3) as the laser beam strikes a moving target. The laser power can be expressed as

$$
P_{\mathrm{F}}=P_{0}\left[1+m \cos \left(\phi_{\mathrm{F}}\right)\right],
$$

where $P_{\mathrm{F}}$ is the laser power under feedback, $P_{0}$ is the laser power without feedback and $m$ is a modulation index. To calculate the phase $\phi_{F}$ in eq. (1) the solver discriminate two regimes of $C$. The first regime is for weak feedback, $C \leq 1.0$, where the right hand side of eq. (1) is monotonic and a unique solution can be found. The second regime is for moderate or high feedback $C \geq 1.0$, and several solutions for eq. (1) exist.

The $m$ parameter in eq.(3) depends on the reflectivity $R_{\text {ext }}$, and influences the amplitude of the fringes. For small displacements (a few half wavelengths) it can be considered as constant. However, for displacements with important amplitudes, $m$ can vary significantly because of variations of the amount of light being coupled back in the cavity. Such variations may be due to Speckle effect or defocusing for example amongst others [29]. In our experiments the optical conditions are set to remain unchanged and in simulations we choose to keep this term constant.

\section{EXPERIMENT AND SIMULATION}

\section{A. Experiment}

A distributed feedback laser diode (ML720J11S) that emits a transverse and longitudinal single mode at $1310 \mathrm{~nm}$ is mounted on an Arroyo Instruments laser mount, including a temperature control driven by a ThorLabs TED 200C. The laser beam is focalized 0.3 meters from the laser source by a ThorLabs C230TMD-C lens. The target is the metal surface of the PI P-753.2CD piezo transducer, driven by a E-665.CR LVPZT-Amplifier. The setup is schematized in Fig. 1. The input voltage for the piezo controller is set to $2.24 V_{\mathrm{pp}}$ which generate 8 interferometric fringes. These fringes are visualized in the first plot in Fig. 2. The oscillation frequency is $8 \mathrm{~Hz}$.

By modulating the piezo transducer at two separate frequencies simultaneously, we are able to produce the effects of the fringe's shape in the OFI signal at small displacement amplitudes. This is achieved by adding a small $7 \mathrm{kHz}$ oscillation with an amplitude of $1 V_{\mathrm{pp}}$ to the $8 \mathrm{~Hz}$ main oscillation. The small oscillation corresponds to a movement of approx. $110 \mathrm{~nm}$ peak to peak. The second plot in Fig. 2 shows the smaller oscillation superposed on of the fringe. By using the piezo amplifier's manual offset, we can also move the mean position the piezo manually in order to set the desired operating point on the fringe.

\section{B. Numerical simulations}

Using Kliese's algorithm [23] we simulate the OFI system's power response. The feedback parameter eq. (2) is empirically set to $C=2.5$ and $\alpha=3$ for an external cavity length of $L_{\text {ext }}=n \lambda_{\text {laser }} / 2 \approx 0.3 \mathrm{~m}$ so that the simulated fringes resemble as much as possible the measured ones.

In the simulations we modify the position of the operating point by modifying $L_{\text {ext }}$. The operating point is best explained as the point on the fringe around which the power response oscillates. We simulate three scenarios where the operating point is

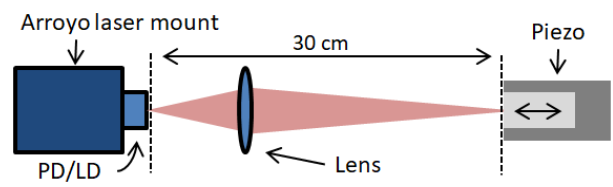

Fig. 1. Schema of experimental setup (not to scale). Pizeo modulated at two separate frequencies simultaneously. 

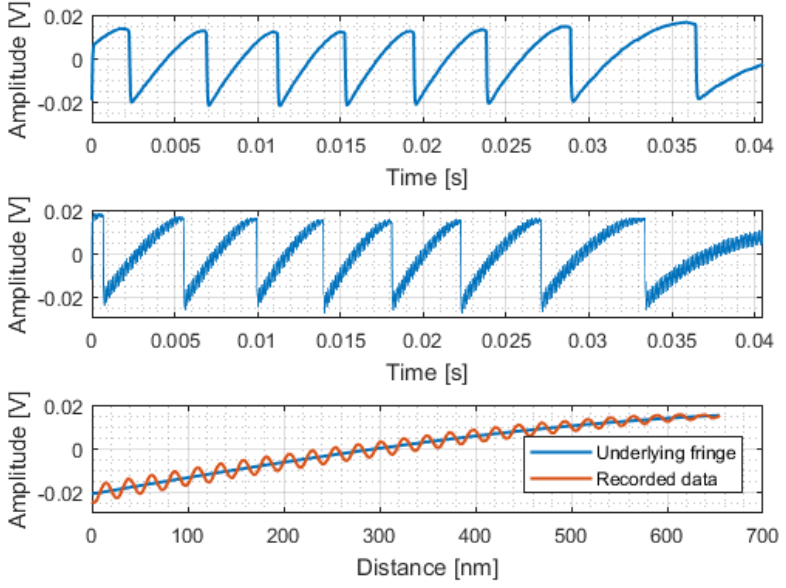

Fig. 2. Experimentally acquired, laser responses measured in Volts. Top: Fringes generated by moving target modulated at $8 \mathrm{~Hz}$ with amplitude $5.02 \mu \mathrm{m}$ zero to peak. Middle: Fringes generated by moving target modulated at $8 \mathrm{~Hz}$ and at $7 \mathrm{kHz}$ with amplitudes $5.02 \mu \mathrm{m}$ and $110 \mathrm{~nm}$ respectively. Bottom: Zoom on fringe number 3 from the left in the middle plot. Fringe with small oscillations on top. The underlying fringe has been approximated by a $3^{r d}$ degree polynomial.

at three different positions, and for each operating point position we simulate a target displacement of amplitude $110 \mathrm{~nm}$ and frequency $7 \mathrm{kHz}$. Figures 3 and 4 shows the output power variation as the target moves back and forth around each operating point.

\section{DISCUSSION}

The simulations presented in Fig. 3 show that the slope $\beta$ of the fringe has an effect on the phase-to-power conversion gain, and thus the amplitude of the detected signal. The slope is calculated by considering the change in laser power per change in external cavity length. It can also be approximated by change in laser power per change in time.

$$
\beta=\frac{\mathrm{d} \cos \left(\phi_{\mathrm{F}}\right)}{\mathrm{d} \phi_{0}} \approx \frac{\mathrm{d} \cos \left(\phi_{\mathrm{F}}\right)}{\mathrm{d} t}
$$

The phase $\phi_{0}=\tau_{\text {ext }} \cdot 2 \pi c / \lambda_{\text {laser }}$ expresses the distance between the laser and the target through $\tau_{\text {ext }} . c$ is the speed of light and $t$ is time. Since the target moves at constant velocity over the range of the fringe, we can approximate $\beta$ by deriving with respect to time $d t$ instead. Simply put, the change in laser power per unit distance is equal to the change of laser power per unit time for a linearly moving target. As we'll see later, this approximation enables us to analyze the fringe slope in the time-domain signals acquired in experiments.

By setting the operating point between positions $A$ and $B$ in Fig. 3 the gain is higher than between positions $C$ and $D$ by a factor 1.47. Furthermore one can estimate the fringe slopes over the parts that produced the interferometric signals by drawing a straight line from position $A$ to $B$ and then from $C$ to $D$. The slope between positions $\mathrm{A}$ and $\mathrm{B}$ is calculated to $\beta=0.3$ whereas the slope from $C$ to $D$ is $\beta=0.2$. In applications such as the one in [6], locking the operation point to the beginning of the fringe would thus be more beneficial when measuring very small changes in the optical path.
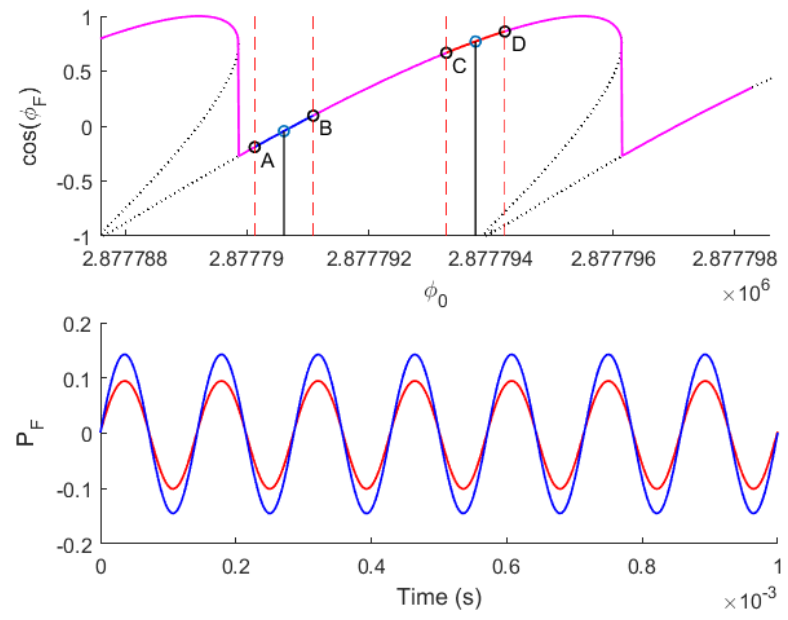

Fig. 3. Simulation of laser power response (arb. units) as function of operating point. Top figure: Black dotted line: Phase development $\cos \left(\phi_{\mathrm{F}}\right)$, Purple line: corresponding fringes, Blue and red lines: part of fringe traveled. Bottom figure: Blue line: Power response between positions $\mathrm{A}$ and B, Red line: Power response between positions $\mathrm{C}$ and $\mathrm{D}$.

By further moving the operating point we can completely distort the output signals as shown in Fig. 4. Positions E and $\mathrm{F}$ have been placed on top of the fringe, right before the discontinuity. The simulation of the oscillation between these two positions confirm comments from [22], where the authors stated that placing the operating point on the top of the fringe would distort their signal.

To further validate the performance of Kliese's algorithm [23] in the sub- $\lambda / 2$ domain we have compared the acquired signal to a simulation, as shown in Fig. 5. In the simulation we replicated the piezo's real movement while setting other experimental
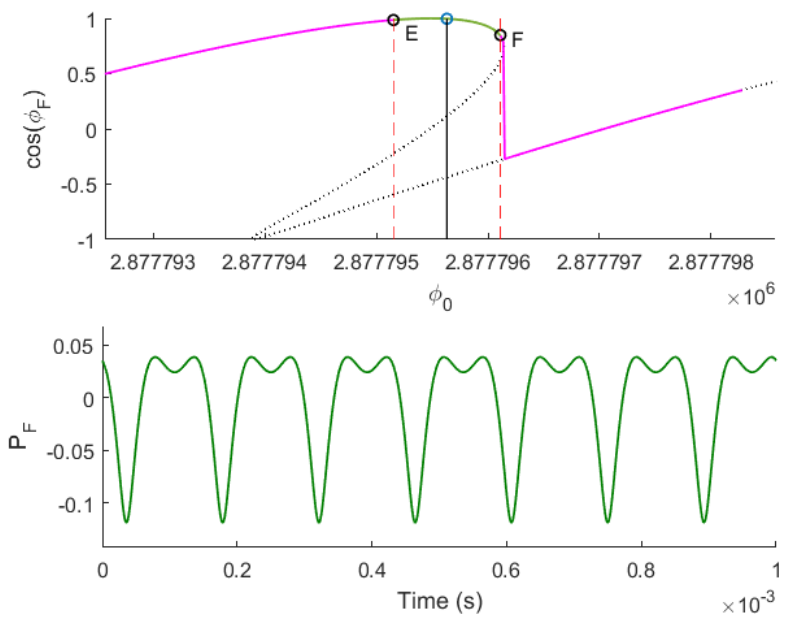

Fig. 4. Simulation of laser power response (arb. units) as function of operating point. Top figure: Black dotted line: Phase development $\cos \left(\phi_{\mathrm{F}}\right)$, Purple line: corresponding fringes, green line: part of fringe traveled. The top of the fringe $\left(\cos \left(\phi_{\mathrm{F}}\right)=1\right)$ is marked with a red spot. Bottom figure: Power response between positions $\mathrm{E}$ and $\mathrm{F}$. 

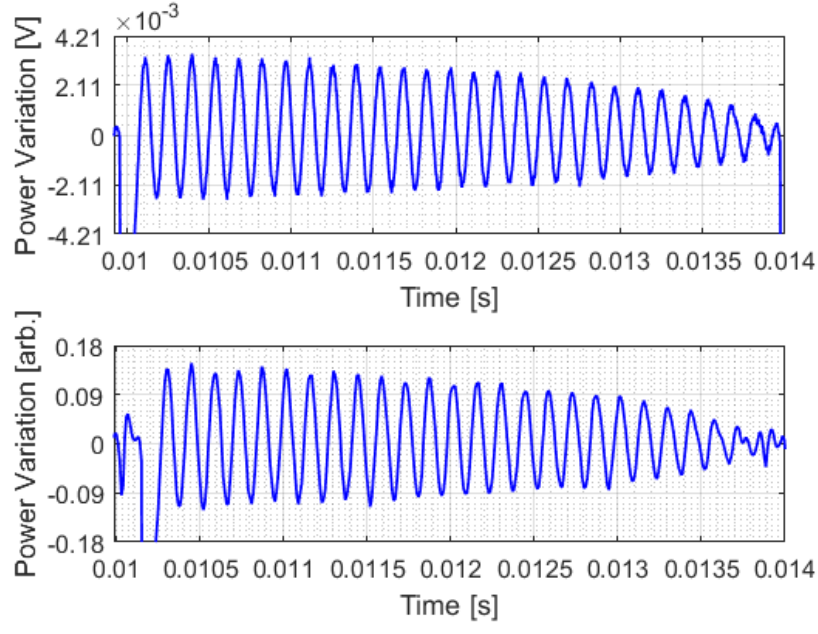

Fig. 5. Comparison of measured interferometric signal (top) and simulation (bottom). The fringes have been removed using a high-pass filter, leaving only the $7 \mathrm{kHz}$ oscillation in the signal. The amplitude of the $7 \mathrm{kHz}$ oscillation is greater at the beginning of the fringe than at the end. The simulation result is remarkably true to the experiment.

parameters such as $L_{\text {ext }}$ and $C$ (described in section 3.A) equal to those in the experiment. Figure. 5 is a zoom on fringe number 3 from the left in Fig. 2. For both the simulated and acquired signal, the fringe's slow amplitude change is removed by a $1^{\text {st }}$ order Butterworth high-pass filter with a cutoff frequency of $6 \mathrm{kHz}$, thus leaving only the $7 \mathrm{kHz}$ component.

Figure 5 offers a good visualization of how well Kliese's algorithm [23] works for sub- $\lambda / 2$ optical path variations as there is clear agreement between the experimental and simulated result. We observe how the amplitude of the $7 \mathrm{kHz}$ oscillation diminishes towards the top of the fringe (towards the right) in both the experiment and in the simulation, followed by a distortion of the signal before the fringe discontinuity.

In order to demonstrate that the signal amplitude of a sub$\lambda / 2$ oscillation is linearly dependent on the slope of the underlying fringe we study the last plot Fig. 2. We have extracted the third fringe in the middle plot, and we have approximated the underlying fringe by a $3^{\text {rd }}$ order polynomial. The $x$-axis is converted to meters in order to reflect the target displacement of a half laser wavelength of $655 \mathrm{~nm}$, which generate one fringe. We study the slope of the fringe, like in the simulations in Figures 3 and 4 , and compare it to the amplitude of the $7 \mathrm{kHz}$ oscillation.

The linear relationship is found by comparing the peak-topeak amplitude variation $\Delta V$ over the full fringe, and the variation of slope $\beta$ of the fringe. The peak to peak amplitude $\Delta V$ is extracted from the bottom plot in Fig 2 using a peak detection algorithm. The fringe slope is computed as the first derivative of the power variation depicted in Fig 2 (bottom). The amplitude and the fringe slope are normalized with regard to their average value over the full fringe $(\langle\beta\rangle$ and $\langle\Delta V\rangle)$.

$$
\beta_{\text {norm }}=\frac{\beta}{<\beta>}
$$

and

$$
V_{\text {norm }}=\frac{\Delta V}{<\Delta V>}
$$
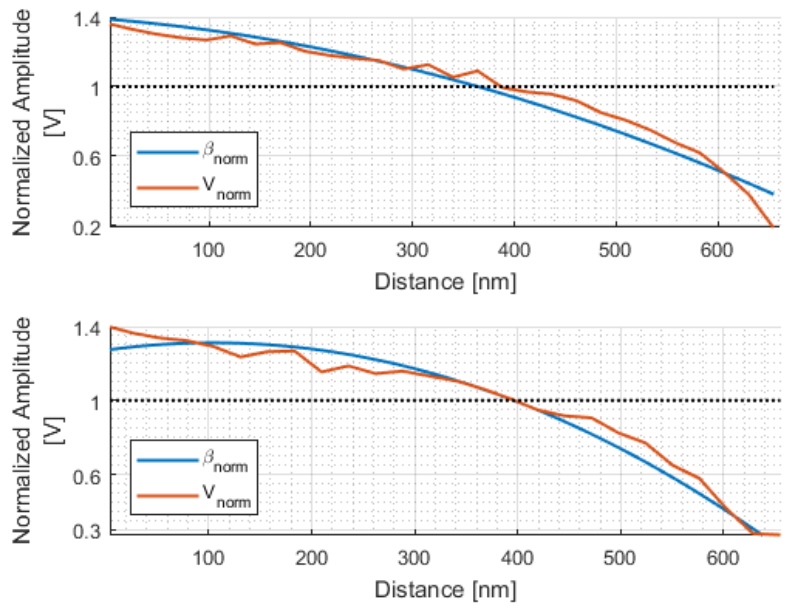

Fig. 6. Evolution of the slope of the fringe and the evolution of the amplitude of the oscillations. Top: Based on signal from experiment (top plot in Fig. 5). Bottom: Based on simulated signal (bottom plot in Fig. 5).

are shown in the first plot Fig. 6. The second plot in the figure shows the result for the simulated signal. The recorded and the simulated signals provide similar results: The signal amplitude decreases at the same rate as the slope of the underlying fringe. Thus we have demonstrated that the relationship between the amplitude of a sub- $\lambda / 2$ signal and the fringe's slope is linear, a result that - to the best of our knowledge - has not been explicitly reported in literature so far.

These results may have have a great impact on measurements of nanometric scale amplitude displacement or using the acousto-optic effect $[30,31]$. By adjusting the operating point on the fringe we believe we can increase the detectors sensitivity for very small displacements well below the laser's half-wavelength. This would also have an impact on acoustic detection using the OFI detection scheme as we would be able to detect lower acoustic powers than before.

Since the linear relationship between the slope of the fringe and the signal amplitude is demonstrated, an interesting solution would be to set the operating point where this slope is at the highest, that is to say in the fringe discontinuity. However, with large coupling coefficient $C$, maintaining such an operating point is difficult while controlling it with an open loop injection current command. Nevertheless, in order to demonstrate the validity of this hypothesis we have composed an experiment in which we degraded the strength of the feedback coupling by defocusing the laser beam. We estimated $C \sim 0.8$ using [23]. We then used the piezo amplifier's manual actuator to shift the mean position of the piezo, and hence the fringe operating point. The piezo was then modulated at $100 \mathrm{~Hz}$ at $3 \mathrm{mV}$ peak to peak which corresponds to an amplitude of $8 \mathrm{~nm}$ peak to peak, which is the smallest stable oscillation we can produce with our system. First, the operating point was set approximately to the middle of the fringe. Then, the operating point was set in the discontinuity.

The first plot in Fig. 7 is an acquisition of the fringes obtained with the coupling factor $C \sim 0.8$. Estimations of the operating point positions for the following acquisitions are marked in black and red. The second plot shows the time-domain acquisition of the laser power variations while the operating point is within the black zone. The third plot shows the time-domain 

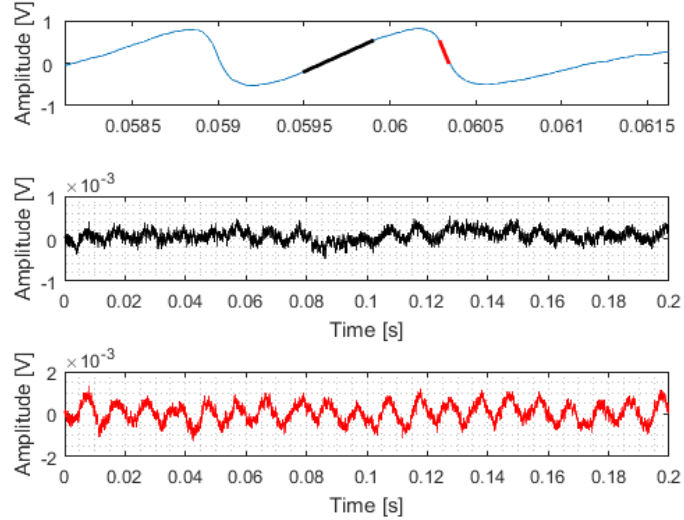

Fig. 7. Top: Shape of fringes used in experiment. Comparison between operating point placed mid-fringe (Middle), and operating point in phase jump (Bottom). A barely detectable displacement signal gains amplitude by clever positioning. Piezo movement: $8 \mathrm{~nm}_{\mathrm{pp}}$. Marked in red and black in top plot: Approximate operating point zones for the two plots.

signal when the operating point is set within the red zone, in the discontinuity. As expected, the amplitude is higher in the last plot. The frequency - domain of the two signals were calculated and analyzed. The peak of the $100 \mathrm{~Hz}$ component of the signal produced mid-fringe had an amplitude of $-98.82 \mathrm{dBV}$ and a noise level of $-107 \mathrm{dBV}$. Thus the peak is only $8.18 \mathrm{~dB}$ above the noise floor. With the operating point positioned in the discontinuity, the amplitude of the $100 \mathrm{~Hz}$ was $-80.93 \mathrm{dBV}, 17.8 \mathrm{~dB}$ above the noise floor of $98.73 \mathrm{dBV}$.

While the $C$ increases it implies towards a steeper and steeper intra-fringe zone. However, a steep slope will limit the dynamic range of the measurement. If a displacement is larger than the intra-fringe zone allows, the operating point will jump to a new stable mode. Further studies of the laser dynamics for higher feedback levels will clarify the limitations and benefits of such a system.

This result opens up for new investigations in the sub- $\lambda / 2$ OFI sensing domain, with several exciting questions to investigate such as; The dynamic range dependency to the feedback conditions; The upper limitation of $C$, and; The determination of an optimal intra-fringe condition that could be achieved while modifying the feedback coupling. One could also imagine a system similar to the one employed in [6] to lock the phase onto the fringe jump, and thus exploit the higher phase-to-power gain factor. Further research shall clarify these questions and further advance the OFI sensing scheme.

\section{CONCLUSION}

In this paper we have shown experimentally and by simulation that the amplitude of a sub- $\lambda / 2$ measurement is linearly dependent on the slope of the underlying fringe. This result has to our knowledge not yet been explicitly presented in the literature. We have validated experimentally the performances of Kliese's algorithm [23] in the sub- $\lambda / 2$ measurement domain by replicating our experiment in simulation and obtaining similar results.

As confirmed by experiments, a simulation of an optical path variation around several positions on the fringe resulted in different output signal amplitudes. The simulation show that a steeper fringe offers a higher gain in the phase to power conversion. A simulation of the piezo's real displacement from the experiment offers similar results to those recorded from the experiment.

Finally we have shown experimentally that placing the operating point in the phase jump, when $C<1$, we can increase the signal amplitude for a small displacement. Exploring this very interesting piste shall provide further understanding of the OFI sensing scheme and its limits. A device such as the one presented in [6] could benefit from this study, by locking the phase at a more suitable position than the middle of the fringe.

\section{ACKNOWLEDGMENTS}

This work has been performed with the LabCom CapIRO - a joint laboratory with the Toulouse INP, The LAAS - CNRS and ACOEM. Grants and funding provided by the Agence Nationale de la Recherche (ANR) and the Association Nationale de la Recherche et de la Technologie (ANRT).

\section{DISCLOSURES}

The authors declare no conflicts of interest.

\section{REFERENCES}

1. S. Donati, "Developing self-mixing interferometry for instrumentation and measurements," Laser \& Photonics Rev. 6, 393-417 (2012).

2. T. Taimre, M. Nikolić, K. Bertling, Y. L. Lim, T. Bosch, and A. D. Rakić, "Laser feedback interferometry: a tutorial on the self-mixing effect for coherent sensing," Adv. Opt. Photonics 7, 570 (2015).

3. C. Bes, G. Plantier, and T. Bosch, "Displacement measurements using a self-mixing laser diode under moderate feedback," IEEE Transactions on Instrumentation Meas. 55, 1101-1105 (2006).

4. I. Milesi, M. Norgia, P. P. Pompilio, C. Svelto, and R. L. Dellaca, "Measurement of local chest wall displacement by a custom self-mixing laser interferometer," IEEE Transactions on Instrumentation Meas. 60, 2894-2901 (2011).

5. A. L. Arriaga, F. Bony, and T. Bosch, "Real-Time Algorithm for Versatile Displacement Sensors Based on Self-Mixing Interferometry," IEEE Sensors J. 16, 195-202 (2016).

6. G. Giuliani, S. Bozzi-Pietra, and S. Donati, "Self-mixing laser diode vibrometer," Meas. Sci. Technol. 14, 24-32 (2003).

7. Z. A. Khan, U. Zabit, O. D. Bernal, M. O. Ullah, and T. Bosch, "Adaptive cancellation of parasitic vibrations affecting a self-mixing interferometric laser sensor," IEEE Transactions on Instrumentation Meas. 66, 332339 (2017).

8. S. Donati and M. Norgia, "Self-Mixing Vibrometer has picometer sensitivity by exploiting the FM Channel," in Conference on Lasers and Electro-Optics, (OSA, San Jose, California, 2018), p. ATu4M.7.

9. M. Norgia, G. Giuliani, and S. Donati, "Absolute Distance Measurement With Improved Accuracy Using Laser Diode Self-Mixing Interferometry in a Closed Loop," IEEE Transactions on Instrumentation Meas. 56, 1894-1900 (2007).

10. L. Rovati, L. Di Cecilia, and S. Cattini, "On the feasibility of absolute distance measurement by using optical-feedback into a superluminescent diode cavity," IEEE Transactions on Instrumentation Meas. pp. 1-1 (2019).

11. M. Veng, J. Perchoux, and F. Bony, "Fringe Disappearance in SelfMixing Interferometry Laser Sensors: Model and Application to the Absolute Distance Measurement Scheme," IEEE Sensors J. pp. 1-1 (2019).

12. G. Plantier, N. Servagent, A. Sourice, and T. Bosch, "Real-time parametric estimation of velocity using optical feedback interferometry," IEEE Transactions on Instrumentation Meas. 50, 915-919 (2001).

13. A. Magnani, A. Pesatori, and M. Norgia, "Real-Time Self-Mixing Interferometer for Long Distances," IEEE Transactions on Instrumentation Meas. 63, 1804-1809 (2014). 
14. S. K. Ozdemir, I. Ohno, and S. Shinohara, "A Comparative Study for the Assessment on Blood Flow Measurement Using Self-Mixing Laser Speckle Interferometer," IEEE Transactions on Instrumentation Meas. 57, 355-363 (2008).

15. M. Norgia, A. Pesatori, and L. Rovati, "Self-mixing laser Doppler: a model for extracorporeal blood flow measurement," in 2010 IEEE Instrumentation \& Measurement Technology Conference Proceedings, (IEEE, Austin, TX, USA, 2010), pp. 304-307.

16. R. Atashkhooei, E. E. Ramírez-Miquet, R. d. C. Moreira, A. Quotb, S. Royo, and J. Perchoux, "Optical Feedback Flowmetry: Impact of Particle Concentration on the Signal Processing Method," IEEE Sensors J. 18, 1457-1463 (2018).

17. V. Contreras, J. Lonnqvist, and J. Toivonen, "Edge filter enhanced self-mixing interferometry," Opt. Lett. 40, 2814-2817 (2015).

18. S. Donati and M. Norgia, "Self-mixing interferometer with a laser diode: Unveiling the fm channel and its advantages respect to the am channel," IEEE J. Quantum Electron. 53, 1-10 (2017).

19. M. Norgia, D. Melchionni, and S. Donati, "Exploiting the fm-signal in a laser-diode smi by means of a mach-zehnder filter," IEEE Photonics Technol. Lett. 29, 1552-1555 (2017).

20. K. Bertling, J. Perchoux, T. Taimre, R. Malkin, D. Robert, A. D. Rakić, and T. Bosch, "Imaging of acoustic fields using optical feedback interferometry," Opt. Express 22, 30346 (2014).

21. P. F. Urgiles Ortiz, J. Perchoux, A. L. Arriaga, F. Jayat, and T. Bosch, "Visualization of an acoustic stationary wave by optical feedback interferometry," Opt. Eng. 57, 1 (2018).

22. A. N. Lukashkin, M. E. Bashtanov, and I. J. Russell, "A self-mixing laser-diode interferometer for measuring basilar membrane vibrations without opening the cochlea," J. Neurosci. Methods 148, 122-129 (2005).

23. R. Kliese, T. Taimre, A. A. A. Bakar, Y. L. Lim, K. Bertling, M. Nikolić, J. Perchoux, T. Bosch, and A. D. Rakić, "Solving self-mixing equations for arbitrary feedback levels: a concise algorithm," Appl. Opt. 53, 3723 (2014).

24. R. Lang and K. Kobayashi, "External optical feedback effects on semiconductor injection laser properties," IEEE J. Quantum Electron. 16, 347-355 (1980)

25. K. Petermann, "Semiconductor Lasers with Optical Feedback," in Laser Diode Modulation and Noise, (Springer Netherlands, Dordrecht, 1988), pp. 250-290.

26. C. Henry, "Theory of the linewidth of semiconductor lasers," IEEE J. Quantum Electron. 18, 259-264 (1982).

27. G. Acket, D. Lenstra, A. D. Boef, and B. Verbeek, "The influence of feedback intensity on longitudinal mode properties and optical noise in index-guided semiconductor lasers," IEEE J. Quantum Electron. 20, 1163-1169 (1984).

28. T. Taimre and A. D. Rakić, "On the nature of Acket's characteristic parameter C in semiconductor lasers," Appl. Opt. 53, 1001 (2014).

29. U. Zabit, O. D. Bernal, and T. Bosch, "Self-Mixing Laser Sensor for Large Displacements: Signal Recovery in the Presence of Speckle," IEEE Sensors J. 13, 824-831 (2013).

30. L. Zipser and H. Franke, "5E-4 Refracto-Vibrometry for Visualizing Ultrasound in Gases, Fluids and Condensed Matter," in 2007 IEEE Ultrasonics Symposium Proceedings, (IEEE, New York, NY, 2007), pp. 395-398.

31. L. Zipser, H.-D. Seelig, and H. Franke, "Refracto-vibrometry for visualizing ultrasound in small-sized channels, cavities and objects," in 2009 IEEE International Ultrasonics Symposium, (IEEE, Rome, Italy, 2009), pp. 2588-2591. 